\title{
THE STRUCTURE OF EXT(A, Z) AND GCH: POSSIBLE CO-MOORE SPACES
}

\author{
PAUL C. EKLOF AND SAHARON SHELAH
}

\begin{abstract}
We investigate what $\operatorname{Ext}(A, \mathbb{Z})$ can be when $A$ is torsion-free and $\operatorname{Hom}(A, \mathbb{Z})=$
0. We thereby give an answer to a question of Golasiński and Gonçalves which asks for the divisible Abelian groups which can be the type of a co-Moore space.
\end{abstract}

\section{INTRODUCTION}

Marek Golasiński and Daciberg Lima Gonçalves have asked which divisible abelian groups $D$ can be the type of a co-Moore space [6] Problem 2.6]. In other words, for which $D$ is there a topological space $X$ such that for some $n \geq 2$, the integral cohomology of $X$ satisfies

$$
H^{i}(X, \mathbb{Z})=\left\{\begin{array}{cc}
D & i=n \\
0 & \text { otherwise }
\end{array}\right.
$$

(cf. [8, pp. 48f]).

This translates, by means of the Universal Coefficient Theorem, into an algebraic question which is of interest in itself: what is the possible structure of $\operatorname{Ext}(A, \mathbb{Z})(=D)$ when $A$ is a torsion-free abelian group such that $\operatorname{Hom}(A, \mathbb{Z})=0$ ?

Date: October 30, 2018.

1991 Mathematics Subject Classification. Primary:20K20, 20K35, 03E35 03E75, 55N99 .

Key words and phrases. co-Moore space, GCH, structure of Ext, rank of Ext, weak diamond .

First author partially supported by NSF DMS 98-03126.

Second author partially supported by NSF DMS 97-04477 and by the German-Israeli Foundation. Publication 717. 
Previous work of Hiller, Huber and Shelah [8] has answered this question under the very strong assumption of Gödel's Axiom of Constructibility (V = L). Here we consider the question under the milder assumption of the Generalized Continuum Hypothesis, GCH, and find weaker restrictions and matching new possibilities.

When $A$ is torsion-free, $\operatorname{Ext}(A, \mathbb{Z})$ is a divisible group and hence isomorphic to

$$
\mathbb{Q}^{\left(\nu_{0}(A)\right)} \oplus \bigoplus_{p \in \mathcal{P}} Z\left(p^{\infty}\right)^{\left(\nu_{p}(A)\right)}
$$

for some cardinals $\nu_{0}(A), \nu_{p}(A)(p \in \mathcal{P}$, the set of primes) which are uniquely determined by $A$ (cf. [5, Chaps. IV, IX]). We want to know what cardinals are possible under the assumption that $\operatorname{Hom}(A, \mathbb{Z})=0$. With regard to the $\nu_{p}(A)$, we have the following lemma of Hiller-Huber-Shelah [8, Prop. 2] (provable in ZFC).

Lemma 0.1. If $A$ is torsion-free and $\operatorname{Hom}(A, \mathbb{Z})=0$, then for every prime $p, \nu_{p}(A)$ is finite or of the form $2^{\mu_{p}}$ for some infinite cardinal $\mu_{p}$.

Regarding $\nu_{0}(A)$, in 8 ] the following is proved assuming $\mathrm{V}=\mathrm{L}$. (The same result is proved in [2] under the weaker hypothesis that every Whitehead group is free). For countable $A$, the result is true in $\mathrm{ZFC}$ (see [3, X11.2.1]).

Proposition 0.2. Assume $V=L$ (or just that every Whitehead group is free). If $A$ is torsion-free and $\operatorname{Hom}(A, \mathbb{Z})=0$, then $\nu_{0}(A)=2^{|A|}$.

Notice that it follows that, under the hypothesis of the Proposition, $\nu_{p}(A) \leq \nu_{0}(A)$ for every prime $p$. Conversely, Hiller-Huber-Shelah prove (in ZFC) that for any cardinals $\nu_{0}, \nu_{p}(p \in \mathcal{P})$ satisfying the conditions that each $\nu_{p}$ is $\leq \nu_{0}$ and is either finite or $2^{\mu_{p}}$ for some infinite $\mu_{p}$, and that $\nu_{0}=2^{\mu_{0}}$ for some infinite $\mu_{0}$, there is a torsion-free group $A$ of cardinality $\mu_{0} \operatorname{such}$ that $\operatorname{Hom}(A, \mathbb{Z})=0, \nu_{0}(A)=\nu_{0}$ and $\nu_{p}(A)=\nu_{p}$ for every $p \in \mathcal{P}$. 
(See [8, Thm. 3(b)].) So the problem of Golasiński and Gonçalves is completely solved under a strong assumption such as $\mathrm{V}=\mathrm{L}$.

Here we are interested in what is possible under the weaker assumption GCH. Our main results are the following two theorems. The first says that if $\nu_{0}(A)$, the (torsionfree) rank of $\operatorname{Ext}(A, \mathbb{Z})$, is less than the value given in Proposition 0.2 then all the $\nu_{p}(A)$ must be as large as possible. (The assumption of GCH is used in the form of the diamond or weak diamond principles it implies.) The second says that all possibilities (for groups $A$ of cardinality $\aleph_{1}$ ) allowed by the first theorem are realized in some model of GCH.

Theorem 0.3. Assume GCH. For any torsion-free group A of uncountable cardinality, if $\operatorname{Hom}(A, \mathbb{Z})=0$ and the rank, $\nu_{0}(A)$, of $\operatorname{Ext}(A, \mathbb{Z})$ is $<2^{|A|}$, then for each prime $p$, the p-rank, $\nu_{p}(A)$, of $\operatorname{Ext}(A, \mathbb{Z})$ is $2^{|A|}$.

We note that, by [10], it is consistent with ZFC + GCH that there are torsion-free groups $A$ of cardinality $\aleph_{1}$ such that the rank of $\operatorname{Ext}(A, \mathbb{Z})$ is $<2^{\aleph_{1}}$ but the $p$-rank of $\operatorname{Ext}(A, \mathbb{Z})$ is also $<2^{\aleph_{1}}$ for some, or all, primes $p$. Of course, in this case (by Theorem [0.3) $\operatorname{Hom}(A, \mathbb{Z})$ must be non-zero. Interestingly, however, the method of [10] can be used to prove the following:

Theorem 0.4. It is consistent with $Z F C+G C H$ that for any cardinal $\rho \leq \aleph_{1}$, there is a strongly $\aleph_{1}$-free group $A$ of cardinality $\aleph_{1}$ such that $\operatorname{Hom}(A, \mathbb{Z})=0$ and the rank of $\operatorname{Ext}(A, \mathbb{Z})$ is $\rho$ (and, by Theorem 0.3 the p-rank of $\operatorname{Ext}(A, \mathbb{Z})$ is $2^{\aleph_{1}}$ for each prime $\left.p\right)$.

Putting together our results with those proved in [6] and [8], we can give a complete answer (assuming GCH) to the question of which divisible groups

$$
D=\mathbb{Q}^{\left(\nu_{0}\right)} \oplus \bigoplus_{p \in \mathcal{P}} Z\left(p^{\infty}\right)^{\left(\nu_{p}\right)}
$$


of cardinality $\leq \aleph_{2}$ are of the form $\operatorname{Ext}(A, \mathbb{Z})$ for some $A$ with $\operatorname{Hom}(A, \mathbb{Z})=0$ :

- $D$ cannot have cardinality $\aleph_{0}$ (cf. [6, Cor. 1.5], 9] Lemma 5]);

- for $D$ of cardinality $\aleph_{1}\left(=2^{\aleph_{0}}\right)$, they are precisely those for which $\nu_{0}=\aleph_{1}$ and each $\nu_{p}$ is either finite or $\aleph_{1}$;

- those $D$ of cardinality $\aleph_{2}\left(=2^{\aleph_{1}}\right)$ which can be proved in ZFC to be of this form are those with $\nu_{0}=\aleph_{2}$ and each $\nu_{p}$ is either finite or $\aleph_{1}$ or $\aleph_{2}$;

- the only other divisible groups $D$ of cardinality $\aleph_{2}$ for which it is consistent with ZFC + GCH that they are of this form are those for which $\nu_{0} \leq \aleph_{1}$ and each $\nu_{p}$ equals $\aleph_{2}$; on the other hand, it is consistent with ZFC + GCH (in particular true in a model of $\mathrm{V}=\mathrm{L})$ that none of these $D$ are of the form $\operatorname{Ext}(A, \mathbb{Z})$ where $\operatorname{Hom}(A, \mathbb{Z})=0$.

By modifying the forcing we can also prove:

Theorem 0.5. It is consistent with $Z F C+G C H$ that there is a non-free strongly $\aleph_{1}$-free group $A$ of cardinality $\aleph_{1}$ such that $\operatorname{Ext}(A, \mathbb{Z})=0$ and $\operatorname{Hom}(A, \mathbb{Z})$ is free. In particular $A$ is a non-reflexive Whitehead group.

In [4] the consistency with ZFC of the existence of such a group was proved using a different forcing (making $2^{\aleph_{0}}>\aleph_{1}$ ), and a weak version of Theorem 0.4 (the case $\rho=0$ ) was also shown consistent with $\mathrm{ZFC}+\neg \mathrm{CH}$.

\section{The P-RANK of ExT}

In this section we will prove Theorem 0.3 Throughout, $A$ will denote a torsion-free group of uncountable cardinality $\kappa$. We will denote the torsion-free rank (resp. $p$-rank) of $\operatorname{Ext}(A, \mathbb{Z})$ by $\nu_{0}(A)\left(\right.$ resp. $\left.\nu_{p}(A)\right)$. The proof will be given in a series of lemmas. 
Lemma 1.1. If $A \cong F / K$ where $F$ is a free group and $K=\oplus_{\alpha<\kappa} K_{\alpha}$ where for all $\alpha<\kappa, \operatorname{Ext}\left(F / K_{\alpha}, \mathbb{Z}\right) \neq 0$, then $\nu_{0}(A)=2^{\kappa}$.

Proof. See [2, Lemma 1.1] or [3, Lemma XII.2.3].

Gregory [7] and Shelah [1] showed that GCH implies diamond for successor cardinals larger than $\aleph_{1}$. Devlin and Shelah [1] proved that weak CH $\left(2^{\aleph_{0}}<2^{\aleph_{1}}\right)$ implies a weak form of diamond at $\aleph_{1}$. In the following, the notation $\Phi_{\lambda}(E)$ means that the weak diamond principle holds for the subset $E$ of $\lambda$ (cf. [3] VI.1.6]).

The invariant $\Gamma_{\lambda, \mathbb{Z}}(A)$ of a group $A$ of cardinality $\lambda$ is defined in [3] p. 352]. We use $\amalg$ to denote disjoint union.

Lemma 1.2. (a) Assume GCH. For any infinite successor cardinal $\lambda, \lambda=\coprod_{\alpha<\lambda} E_{\alpha}$ where for each $\alpha<\lambda, \Phi_{\lambda}\left(E_{\alpha}\right)$ holds.

(b) If $\Gamma_{\lambda, \mathbb{Z}}(A) \supseteq \tilde{E}$ and $\Phi_{\lambda}(E)$ holds, then $\operatorname{Ext}(A, \mathbb{Z}) \neq 0$.

Proof. (a) See [7, 11], 1] and 3, VI.1.10]. (b) See [1] and 3, XII.1.7].

Lemma 1.3. Assume GCH. Suppose that $A$ is the union of a continuous chain of subgroups $\left(A_{\mu}: \mu<\kappa\right)$ of cardinality $<\kappa$ such that for all $\mu<\kappa, A_{\mu+1} / A_{\mu}$ is countable and not free. Then $\nu_{0}(\kappa)=2^{\kappa}$.

Proof. (cf. [2, Thm. 2.14]) By [3] XII.1.4] we can assume that $A=F / K$ where $F=\oplus_{\beta<\kappa} F_{\beta}$ is a free group and $K=\oplus_{\beta<\kappa} K_{\beta}$ such that for every $\mu<\kappa, A_{\mu}=$ $\oplus_{\beta<\mu} F_{\beta} / \oplus_{\beta<\mu} K_{\beta}$, and hence $A_{\mu+1} / A_{\mu} \cong \oplus_{\beta \leq \mu} F_{\beta} /\left(\oplus_{\beta<\mu} F_{\beta}+K_{\mu}\right)$. Let us consider first the case where $\kappa$ is a successor cardinal. By Lemma 1.2 (a), $\kappa=\coprod_{\alpha<\kappa} E_{\alpha}$ where for each $\alpha<\kappa, \Phi_{\kappa}\left(E_{\alpha}\right)$ holds. Now write $K=\oplus_{\alpha<\kappa} K_{\alpha}^{\prime}$ where $K_{\alpha}^{\prime}=\oplus_{\beta \in E_{\alpha}} K_{\beta}$. Then for all $\alpha<\kappa, \Gamma_{\kappa, \mathbb{Z}}\left(F / K_{\alpha}^{\prime}\right) \supseteq \tilde{E}_{\alpha}$ because $F / K_{\alpha}^{\prime}=\bigcup_{\mu<\kappa} H_{\mu}$ where $H_{\mu}=\left(\oplus_{\beta<\mu} F_{\beta}+K_{\alpha}^{\prime}\right) / K_{\alpha}^{\prime}$ 
and hence for $\mu \in E_{\alpha}, H_{\mu+1} / H_{\mu} \cong \oplus_{\beta \leq \mu} F_{\beta} /\left(\oplus_{\beta<\mu} F_{\beta}+K_{\mu}\right) \cong A_{\mu+1} / A_{\mu}$ which is countable and non-free, and hence not a Whitehead group. Therefore, by Lemma 1.2(b), $\operatorname{Ext}\left(F / K_{\alpha}^{\prime}, \mathbb{Z}\right) \neq 0$. Finally, apply Lemma 1.1

Now suppose $\kappa$ is a limit cardinal; then $\kappa=\sup \left\{\kappa_{i}: i<\operatorname{cof}(\kappa)\right\}$ where for each $i<\operatorname{cof}(\kappa), \kappa_{i}$ is a successor cardinal $>\sup \left\{\kappa_{j}: j<i\right\}$. Let $S_{i}=\kappa_{i}-\bigcup\left\{\kappa_{j}: j<i\right\}$; so $S_{i}$ is a set of cardinality $\kappa_{i}$ and $\kappa=\coprod_{i<\operatorname{cof}(\kappa)} S_{i}$. By Lemma 1.2(a), $S_{i}=\coprod_{\alpha<\kappa_{i}} E_{\alpha}^{i}$ where for each $\alpha<\kappa_{i}, \Phi_{\kappa_{i}}\left(E_{\alpha}^{i}\right)$ holds. Let $K_{\alpha}^{i}=\oplus\left\{K_{\beta}: \beta \in E_{\alpha}^{i}\right\}$, so $K=\bigoplus_{i<\operatorname{cof}(\kappa)} \oplus_{\alpha<\kappa_{i}} K_{\alpha}^{i}$. If we can show that $\operatorname{Ext}\left(F / K_{\alpha}^{i}, \mathbb{Z}\right) \neq 0$ for all $\alpha$ and $i$, then we will be done by Lemma 1.1 Since $F / K_{\alpha}^{i}$ contains $\left(\oplus_{\beta \in S_{i}} F_{\beta}\right) / K_{\alpha}^{i}$ it is enough to prove that $\operatorname{Ext}\left(\left(\oplus_{\beta \in S_{i}} F_{\beta}\right) / K_{\alpha}^{i}, \mathbb{Z}\right) \neq$ 0 . But this is the case by Lemma 1.2 (b) because $\left(\oplus_{\beta \in S_{i}} F_{\beta}\right) / K_{\alpha}^{i}$ is a group of cardinality $\kappa_{i}$ satisfying $\Gamma_{\kappa_{i}, \mathbb{Z}}\left(\left(\oplus_{\beta \in S_{i}} F_{\beta}\right) / K_{\alpha}^{i}\right) \supseteq \tilde{E}_{\alpha}^{i}$ and $\Phi_{\kappa}\left(E_{\alpha}^{i}\right)$ holds.

Lemma 1.4. If $A$ contains a pure free subgroup $B$ of cardinality $\kappa$ and $\operatorname{Hom}(A, \mathbb{Z})=0$, then for every prime $p, \nu_{p}(A)=2^{\kappa}$.

Proof. Since $B / p B$ is isomorphic to a subgroup of $A / p A$, the dimension of $A / p A$ as a vector space over $\mathbb{Z} / p \mathbb{Z}$ is $\kappa$. From the exact sequence

$$
0=\operatorname{Hom}(A, \mathbb{Z}) \rightarrow \operatorname{Hom}(A, \mathbb{Z} / p \mathbb{Z}) \rightarrow \operatorname{Ext}(A, \mathbb{Z}) \stackrel{p_{*}}{\rightarrow} \operatorname{Ext}(A, \mathbb{Z})
$$

it follows that $\nu_{p}(A)$ equals the dimension of the kernel of $p_{*}$; but this kernel is $\operatorname{Hom}(A, \mathbb{Z} / p \mathbb{Z}) \cong$ $\operatorname{Hom}(A / p A, \mathbb{Z} / p \mathbb{Z})$, which clearly has dimension $2^{\kappa}$.

Finally we have

Lemma 1.5. Assume $G C H$. If $\nu_{0}(A)<2^{\kappa}$, then $A$ contains a pure free subgroup $B$ of cardinality $\kappa$. 
Proof. First we claim that every subset of $A$ of cardinality $<\kappa$ is contained in a subgroup $C$ of cardinality $<\kappa$ such that $A / C$ is $\aleph_{1}$-free. If not, then $A$ contains a subgroup $A_{0}$ of cardinality $<\kappa$ such that for every subgroup $C$ of cardinality $<\kappa$ containing $A_{0}$, there is a subgroup $C^{\prime}$ of $A$ containing $C$ such that $C^{\prime} / C$ is countable and not free. It follow easily that $A$ is the union of a continuous chain of subgroups $\left(A_{\alpha}: \alpha<\kappa\right)$ each of cardinality $<\kappa$ such that for all $\alpha<\kappa, A_{\alpha+1} / A_{\alpha}$ is countable and not free. But then by Lemma 1.3 $\nu_{0}(A)=2^{\kappa}$, which is a contradiction.

Now let $Y \subseteq A$ be maximal with respect to the property that $Y$ is a basis of a pure free subgroup of $A$. By Lemma 1.4 it suffices to show that $Y$ has cardinality $\kappa$. If not, let $C$ be a subgroup of $A$ containing $Y$ and of cardinality $<\kappa$ such that $A / C$ is $\aleph_{1}$-free. If $C^{\prime} / C$ is a countable, pure and non-zero subgroup of $A / C$; then $C^{\prime} / C$ is free, $C^{\prime}$ is pure in $A$ and $C^{\prime}=C \oplus D$ where $D$ is countable, free and non-zero. Choosing an element $d$ of a basis of $D$, we see that $Y \cup\{d\}$ contradicts the maximality of $Y$.

\section{TheOREM 0.4 THE BASICS}

We now embark on the proof of Theorem 0.4 which will occupy this and the next two sections. Throughout $\rho$ will be a fixed cardinal $\leq \aleph_{1}$ and $S$ will be a stationary and co-stationary subset of $\omega_{1}$ consisting of limit ordinals.

We begin by defining a group $A=A(e, a)$ which depends on two parameters, functions $e$ and $a$. The function $e$ is a function from $S \times \omega$ to the primes such that for all $\delta \in S$, $e(\delta, \cdot)$ is a strictly increasing function of $\omega$. The function $a$ is a function on $S \times \omega$ such that for every $\delta \in S$ and $n \in \omega, a(\delta, n)$ is a finite non-empty subset of $\delta$ such that $\max a(\delta, n+1)>\max a(\delta, n)$ and $\sup \{\max a(\delta, n): n \in \omega\}=\delta$. The functions $e$ and

$a$ that we will use will be generic, so $A$ will be defined in a generic extension of the 
universe; we will then construct a further forcing extension in which $A$ has the desired properties.

Let $F$ be the free abelian group with basis

$$
\left\{x_{\nu}: \nu \in \omega_{1}\right\} \cup\left\{z_{\delta, n}: \delta \in S, n \in \omega\right\} .
$$

Let $K$ be the subgroup of $F$ generated by $\left\{w_{\delta, n}: \delta \in S, n \in \omega\right\}$ where

$$
w_{\delta, n}=e(\delta, n) z_{\delta, n+1}-z_{\delta, 0}+\sum_{\nu \in a(\delta, n)} x_{\nu}
$$

In fact, $\left\{w_{\delta, n}: \delta \in S, n \in \omega\right\}$ is easily seen to be a basis of $K$. Let $A=F / K$. Then clearly $A$ is an abelian group of cardinality $\aleph_{1}$. Notice that because the right-hand side of (2.1) is 0 in $A$, we have for each $\delta \in S$ and $n \in \omega$ the following relations in $A$ :

$$
e(\delta, n) z_{\delta, n+1}=z_{\delta, 0}-\sum_{\nu \in a(\delta, n)} x_{\nu}
$$

Here, and occasionally in what follows, we abuse notation and write, for example, $z_{\delta, n}$ instead of $z_{\delta, n}+K$ for an element of $A$. For each $\alpha<\omega_{1}$, let $A_{\alpha}$ be the subgroup of $A$ generated by

$$
\left\{x_{\nu}: \nu<\alpha\right\} \cup\left\{z_{\delta, n}: \delta \in S \cap \alpha, n \in \omega\right\} .
$$

Then, by (2.2), for each $\delta \in S, z_{\delta, 0}+A_{\delta}$ is non-zero and divisible in $A_{\delta+1} / A_{\delta}$ by infinitely many primes. Thus $A_{\delta+1} / A_{\delta}$ is not free. Moreover, because $A_{\delta+1} / A_{\delta}$ is not free for stationarily many $\delta \in \omega_{1}, A$ is not free (cf. [3, IV.1.7]).

The definition of $\operatorname{Ext}(A, \mathbb{Z})$ that is most convenient for our purposes is that it is $\operatorname{Hom}(K, \mathbb{Z}) / \operatorname{Hom}(F, \mathbb{Z})$ where $\operatorname{Hom}(F, \mathbb{Z})$ stands for the $\operatorname{subgroup~of~} \operatorname{Hom}(K, \mathbb{Z})$ consisting of those homomorphisms which extend to $F$. We shall abuse notation and refer to homomorphisms from $K$ to $\mathbb{Z}$ as elements of $\operatorname{Ext}(A, \mathbb{Z})$ when, strictly speaking, we should 
refer to the coset $\bmod \operatorname{Hom}(F, \mathbb{Z})$ of the homomorphism. A homomorphism $\varphi: K \rightarrow \mathbb{Z}$ is a torsion element of the group $\operatorname{Ext}(A, \mathbb{Z})$ if and only if there is a homomorphism $\psi: F \rightarrow \mathbb{Z}$ and a non-zero integer $d$ such that $\varphi=d \psi \uparrow K$. Otherwise, $\varphi$ is a torsion-free element of $\operatorname{Ext}(A, \mathbb{Z})$.

We now define the forcing extension in which $A$ will be defined using generic data. Besides the generic functions $e$ and $a$ we are going to define generically $\rho$ homomorphisms $\varphi_{s}(s<\rho)$ from $K$ to $\mathbb{Z}$ which will guarantee that the (torsion-free) $\operatorname{rank}$ of $\operatorname{Ext}(A, \mathbb{Z})$ is at least $\rho$. We begin with a model $V$ of ZFC where GCH holds, choose $S \in V$ to be a stationary and co-stationary subset of $\omega_{1}$, and define a poset as follows:

Definition 2.1. Let $Q_{0}$ be the set of all tuples $q$ such that for some $\delta_{0}<\omega_{1}, q=$ $\left\langle e^{q}, a^{q}, f_{s}^{q}: s<\rho \cap \delta_{0}\right\rangle$ and for all $\delta \in \delta_{0} \cap S:$

- $e^{q}(\delta, \cdot): \omega \rightarrow\{p \in \mathbb{Z}: p$ is prime $\}$ and is strictly increasing;

- $a^{q}(\delta, \cdot)$ is a function on $\omega$ such that for all $n \in \omega, a^{q}(\delta, n)$ is a finite non-empty subset of $\delta$ such that $\max a^{q}(\delta, n)<\max a(\delta, n+1)$ and $\sup \left\{\max a^{q}(\delta, n): n \in\right.$ $\omega\}=\delta ;$

- for each $s<\rho \cap \delta_{0}, f_{s}^{q}$ is a function from $\left\{w_{\delta, n}: \delta \in \delta_{0} \cap S, n \in \omega\right\}$ to $\mathbb{Z}$.

We shall refer to $\delta_{0}$ as $\operatorname{dom}(q)$. The partial ordering of $Q_{0}$ is defined by: $q_{1} \leq q_{2}$ if and only if $q_{1} \subseteq q_{2}$; note that we follow the convention that stronger conditions are larger. It is easy to see that for any $\gamma \in \omega_{1},\left\{q \in Q_{0}: \gamma \subseteq \operatorname{dom}(q)\right\}$ is dense in $Q_{0}$. Clearly $Q_{0}$ is $\omega$-closed and satisfies the $\aleph_{2}$-chain condition, so GCH is preserved.

Let $G_{1}$ be $Q_{0}$-generic and in $V\left[G_{1}\right]$ let $A=A(e, a)$ be the group constructed as above with the generic data $e=\cup\left\{e^{q}: q \in G_{1}\right\}$ and $a=\cup\left\{a^{q}: q \in G_{1}\right\}$. Let $\varphi_{s}$ be the homomorphism: $K \rightarrow \mathbb{Z}$ which on the basis $\left\{w_{\delta, n}: \delta \in S, n \in \omega\right\}$ is given by 
$\cup\left\{f_{s}^{q}: q \in G_{1}\right\}$; then $\left\{\varphi_{s}: s<\rho\right\}$ is a linearly independent subset of $\operatorname{Ext}(A, \mathbb{Z})$. Thus the torsion-free rank of $\operatorname{Ext}(A, \mathbb{Z})$ is at least $\rho$ (i.e., $\nu_{0}(A) \geq \rho$ ). However, in $V\left[G_{1}\right]$ the rank will be larger; so we do an iterated forcing to eliminate torsion-free elements of $\operatorname{Ext}(A, \mathbb{Z})$ which are not in the $\mathbb{Q}$-vector space generated by $\left\{\varphi_{s}: s<\rho\right\}$.

We begin by defining the basic forcing that we will iterate.

Definition 2.2. Given a homomorphism $\psi: K \rightarrow \mathbb{Z}$, let $Q_{\psi}$ be the poset of all functions $q$ into $\mathbb{Z}$ such that for some successor ordinal $\alpha \in \omega_{1}$, the domain of $q$ is $\left\{z_{\delta, k}: \delta \in\right.$ $\alpha \cap S, k \in \omega\} \cup\left\{x_{\nu}: \nu<\alpha\right\}$ and for all $\delta \in \alpha \cap S$ and $k \in \omega$

$$
\psi\left(w_{\delta, k}\right)=e(\delta, k) q\left(z_{\delta, k+1}\right)-q\left(z_{\delta, 0}\right)+\sum_{\nu \in a(\delta, k)} q\left(x_{\nu}\right)
$$

(Compare with (2.1)). The partial ordering on $Q_{\psi}$ is inclusion.

In an abuse of notation, if the domain of $q$ is $\left\{z_{\delta, n}: \delta \in \alpha \cap S, n \in \omega\right\} \cup\left\{x_{\nu}: \nu<\alpha\right\}$, we shall write $\operatorname{dom}(q)=\alpha$.

Lemma 2.3. For every $\alpha \in \omega_{1}$ and every $q \in Q_{\psi}$, there exists $q^{\prime} \in Q$ such that $q \leq q^{\prime}$ and $\operatorname{dom}\left(q^{\prime}\right) \geq \alpha$.

Proof. Let $\operatorname{dom}(q)=\beta$; without loss of generality, $\beta<\alpha$. Enumerate $\{\delta \in S: \beta \leq \delta<$ $\alpha\}$ in an $\omega$-sequence $\left\langle\delta_{k}: k \in \omega\right\rangle$ and define by induction on $k$ the values $q\left(z_{\delta_{k}, n}\right)$ and $q\left(x_{\nu}\right)$ so that (2.4) holds; in fact, we can do this so that $q\left(z_{\delta_{k}, n}\right)=0$ for sufficiently large $n$ because for sufficiently large $n, q\left(x_{\max \left(a\left(\delta_{k}, n\right)\right)}\right)$ has not previously been defined, so we can choose it to make (2.4) true. 
Now $P=\left\langle P_{i}, \dot{Q}_{i}: 0 \leq i<\omega_{2}\right\rangle$ is defined to be a countable support iteration of length $\omega_{2}$ so that for every $i \geq 1, \Vdash_{P_{i}} \dot{Q}_{i}=Q_{\dot{\psi}_{i}}$ whenever $\Vdash_{P_{i}}$ " $\dot{\psi}_{i}: K \rightarrow \mathbb{Z}$ is a torsionfree element of $\operatorname{Ext}(A, \mathbb{Z})$ independent of $\left\{\varphi_{s}: s<\rho\right\} " ;$ otherwise, $\Vdash_{P_{i}} \dot{Q}_{i}=0$. The enumeration of names $\left\{\dot{\psi}_{i}: 1 \leq i<\omega_{2}\right\}$ is chosen so that if $G$ is $P$-generic and $\psi \in V[G]$ is a homomorphism: $K \rightarrow \mathbb{Z}$, then for some $i \geq 1, \dot{\psi}_{i}$ is a name for $\psi$ in $V^{P_{i}}$.

Then $P$ is proper, $\left(\omega_{1}-S\right)$-complete (so adds no new $\omega$-sequences) and satisfies the $\aleph_{2}$-chain condition. Moreover, in $V[G]$ every torsion-free element of $\operatorname{Ext}(A, \mathbb{Z})$ is dependent on $\left\{\varphi_{s}: s<\rho\right\}$ so $\nu_{0}(A) \leq \rho$. The proof that $\nu_{0}(A) \geq \rho$ is the same as the main argument in [10]: note that though the first forcing, $Q_{0}$, is not quite the same here (because of the needs of the following lemma), the proof in [10] is still valid.

It remains to prove that, in $V[G], \operatorname{Hom}(A, \mathbb{Z})=0$. Let $G_{\nu}=\{p\lceil\nu: p \in G\}$, so that $G_{\nu}$ is $P_{\nu}$-generic. First we prove:

Lemma 2.4. $\operatorname{Hom}(A, \mathbb{Z})^{V\left[G_{1}\right]}=0$

Proof. By equation (2.2), if $h \in \operatorname{Hom}(A, \mathbb{Z})$ and $h\left(x_{\mu}\right)=0$ for all $\mu \in \omega_{1}$, then $h$ is identically zero. So suppose, to obtain a contradiction, that there exists a $Q_{0}$-name $\dot{h}$ and $r_{0} \in G_{1}$ such that

$$
r_{0} \Vdash \dot{h} \in \operatorname{Hom}(A, \mathbb{Z}) \wedge \dot{h}\left(x_{\mu}\right)=m
$$

for some $\mu \in \omega_{1}$ and some non-zero integer $m$. Choose a strictly increasing sequence of primes $\left(d_{n}: n \in \omega\right)$ all larger than $m$. Choose recursively an increasing chain $\left\{r_{\nu}\right.$ : $\left.\nu \in \omega_{1}\right\}$ of elements of $Q_{0}$ such that if $\alpha_{\nu}=\operatorname{dom}\left(r_{\nu}\right)$, then $\mu<\alpha_{1}$ and for all $\nu$, $\nu \leq \alpha_{\nu}<\alpha_{\nu+1}$ and for some $c_{\nu} \in \mathbb{Z}, r_{\nu+1} \Vdash \dot{h}\left(x_{\alpha_{\nu}}\right)=c_{\nu}$. Moreover, for all limit $\sigma, r_{\sigma}$ is the union of $\left\{r_{\tau}: \tau<\sigma\right\}$, so $\operatorname{dom}\left(r_{\sigma}\right)=\sup \left\{\alpha_{\tau}: \tau<\sigma\right\}$. 
Then, since $S$ is stationary and $\left\{\sigma: \alpha_{\sigma}=\sigma\right\}$ is a club, there is a limit ordinal $\delta$ such that $\operatorname{dom}\left(r_{\delta}\right)=\alpha_{\delta}=\delta \in S$. Choose a strictly increasing sequence $\left(\alpha_{\nu_{n}}: n \in \omega\right)$ whose supremum is $\delta$. Choose a bijection $g: \omega \rightarrow \mathbb{Z}$. For each $n \in \omega$, let $a_{n}=\left\{\alpha_{\nu_{n}}\right\}$ if $d_{n} \rtimes g(n)-c_{\nu_{n}}$ and otherwise $a_{n}=\left\{\alpha_{\nu_{n}}, \mu\right\}$, in which case $d_{n} \rtimes g(n)-c_{\nu_{n}}-m$. There exists $r^{*} \in Q_{0}$ such that $r^{*} \geq r_{\delta}$ and for all $n \in \omega$

$$
r^{*} \Vdash e(\delta, n)=d_{n} \wedge a(\delta, n)=a_{n} .
$$

We obtain a contradiction by considering any generic $G^{*}$ with $r^{*} \in G^{*}$ : indeed, in $V[G]$ we have $h\left(z_{\delta, 0}\right)=g(n)$ for some $n \in \omega$ but also $e(\delta, n) h\left(z_{\delta, n+1}\right)=h\left(z_{\delta, 0}\right)-\sum_{j \in a_{n}} h\left(x_{j}\right)$, which is a contradiction of the choice of $a_{n}$.

We conclude this section with a simple lemma.

Lemma 2.5. Any homomorphism $f$ from $F$ to $\mathbb{Z}$ is completely determined by $f \uparrow\left\{x_{\nu}\right.$ : $\left.\nu \in \omega_{1}\right\} \cup K$.

Proof. This follows from (2.1), since for any $\delta$ and any integers $\left\langle c_{n}: n \in \omega\right\rangle$, there is at most one integral solution to the equations

$$
\left\{e(\delta, n) f\left(z_{\delta, n+1}\right)-f\left(z_{\delta, 0}\right)=c_{n}: n \in \omega\right\}
$$

in the unknowns $f\left(z_{\delta, n}\right)(n \in \omega)$.

\section{3. $\operatorname{Hom}(A, \mathbb{Z})=0$}

In this section and the next we will prove that $\operatorname{Hom}(A, \mathbb{Z})$ remains zero even after our iterated forcing. Let $h \in \operatorname{Hom}(A, \mathbb{Z})^{V[G]}$; then $h \in V\left[G_{i}\right]$ for some $i<\omega_{2}$ since $P$ satisfies the $\aleph_{2}$-chain condition. We shall prove by induction on $i$ that any $h \in \operatorname{Hom}(A, \mathbb{Z})^{V\left[G_{i}\right]}$ belongs to $V\left[G_{1}\right]$ and hence is zero. Let $q_{*} \in G_{i}$ such that $q_{*} \Vdash \dot{h} \in \operatorname{Hom}(A, \mathbb{Z})$. 
Throughout this and the next section, we fix the notations $h, i$, and $q_{*}$. Let $\tilde{P}_{i}$ denote the dense subset of $P_{i}$ consisting of conditions $q$ such that there is an ordinal $\delta$ such that for all $\alpha \in \operatorname{dom}(q), q(\alpha)$ belongs to $V$ and $\operatorname{dom}(q(\alpha))=\delta$. If $q \in \tilde{P}_{i}$, we will write $\operatorname{dom}(q)=\delta$ if $\operatorname{dom}(q(\alpha))=\delta$ for all $\alpha \in \operatorname{dom}(q)$.

Definition 3.1. For any $q \in \tilde{P}_{i}$ and any $0<\alpha<i$, let $\operatorname{Pos}_{\alpha}(q)$ be the set of all sequences of integers $\left\langle c^{0}, c^{1}, \ldots, c^{2 m-2}, c^{2 m-1}\right\rangle$ such that for arbitrarily large $\zeta \in \omega_{1}$ there are $r_{0}, \ldots, r_{m-1} \in \tilde{P}_{i}$ each stronger than $q$ and such that $r_{0}\left\lceil\alpha=\ldots=r_{m-1}\lceil\alpha\right.$ and for $\ell=0, \ldots, m-1, r_{\ell}(\alpha)\left(x_{\zeta}\right)=c^{2 \ell}$ and $r_{\ell} \Vdash^{P_{i}} \dot{h}\left(x_{\zeta}\right)=c^{2 \ell+1}$.

Since $\operatorname{Pos}_{\alpha}(q)$ decreases as $q$ increases, we can assume that $q_{*}$ is such that: if $i$ has cofinality $\omega_{1}$ or $i$ is a successor, then there is $\alpha_{*}<i$ such that

$$
\operatorname{Pos}_{\alpha_{*}}\left(q_{*}\right)=\operatorname{Pos}_{\alpha}(q)
$$

whenever $\alpha_{*} \leq \alpha<i$ and $q \geq q_{*}$, and if $i$ has cofinality $\omega$, then for arbitrarily large $\alpha<i$

$$
\operatorname{Pos}_{\alpha}\left(q_{*}\right)=\operatorname{Pos}_{\alpha}(q)
$$

whenever $q \geq q_{*}$ (cf. [10] E1, p. 77]). (Note that if $i$ has cofinality $\omega$, we can recursively define $q_{*}\left(\alpha_{n}\right)$ on a sequence $\left(\alpha_{n}: n \in \omega\right)$ approaching $i$ so that the second displayed identity holds.)

We shall say that $\alpha$ is good if the appropriate (depending on the cofinality of $i$ ) displayed identity holds for $\alpha$. We assert:

Claim 3.2. There is a good $\alpha$ such that:

(a) for any $\left\langle c^{0}, c^{1}, c^{0}, c^{2}\right\rangle \in \operatorname{Pos}_{\alpha}\left(q_{*}\right), c^{1}=c^{2}$; 
(b) for any $\left\langle c^{0}, c^{1}, c^{2}, c^{3}, c^{4}, c^{5}\right\rangle \in \operatorname{Pos}_{\alpha}\left(q_{*}\right),\left(c^{0}, c^{1}\right),\left(c^{2}, c^{3}\right)$, and $\left(c^{4}, c^{5}\right)$ lie on a straight line, i.e., there are rational numbers $d_{1}, d_{2}$ such that $c^{2 \ell+1}=d_{1} c^{2 \ell}+d_{2}$ for $\ell=0,1,2$

(c) for any $\left\langle c^{0}, c^{1}, c^{2}, c^{3}\right\rangle,\left\langle c^{4}, c^{5}, c^{6}, c^{7}\right\rangle \in \operatorname{Pos}_{\alpha}\left(q_{*}\right)$ with $c^{2} \neq c^{0}$ and $c^{6} \neq c^{4}$, we have

$$
\frac{c^{3}-c^{1}}{c^{2}-c^{0}}=\frac{c^{7}-c^{5}}{c^{6}-c^{4}}
$$

Assuming the Claim we will finish the proof. As motivation for the following argument, consider a simple example.

Example 3.3. Suppose that for some forcing $P$ and every $\zeta \in \omega_{1}$ there are $P$-names $\dot{n}_{\zeta}$ and $\dot{m}_{\zeta}$ for integers such that for no integers $c_{0}, c_{1}$ and $c_{2}$ with $c_{1} \neq c_{2}$ is it possible to have arbitrarily large $\zeta \in \omega_{1}$ for which there are P-generic extensions $V\left[G_{1}\right]$ and $V\left[G_{2}\right]$ with $V\left[G_{\ell}\right] \models " \dot{n}_{\zeta}=c_{0} \wedge \dot{m}_{\zeta}=c_{\ell}$ " for $\ell=1,2$. Then there is a function $f \in V$ and $\zeta_{*} \in \omega_{1}$ such that $\Vdash^{P} \forall \zeta \geq \zeta_{*}\left(\dot{m}_{\zeta}=f\left(\zeta, \dot{n}_{\zeta}\right)\right)$. Note that $f$ may be a function of $\zeta$; e.g., we could have arbitrarily large $\zeta$ for which $\Vdash^{P} \dot{m}_{\zeta}=f_{0}\left(\dot{n}_{\zeta}\right)$ and arbitrarily large $\zeta$ for which $\Vdash^{P} \dot{m}_{\zeta}=f_{1}\left(\dot{n}_{\zeta}\right)$.

We work in $V\left[G_{\alpha}\right]$. Let $\dot{\varphi}_{\alpha}$ be a $Q_{\alpha}$-name for the generic object given by $Q_{\alpha}$, if $Q_{\alpha} \neq 0$, and otherwise $\dot{\varphi}_{\alpha}$ is a name for the zero function. By assumption (a), there is a $\zeta_{*} \in \omega_{1}$ and a function $f \in V$ such that

$$
q_{*} \Vdash^{P_{i} / G_{\alpha}} \forall \zeta \geq \zeta_{*}\left[\dot{h}\left(x_{\zeta}\right)=f\left(\zeta, \dot{\varphi}_{\alpha}\left(x_{\zeta}\right)\right) .\right.
$$


Moreover, by (b) and (c), there is a $\gamma^{*} \in \omega_{1}, d_{1} \in \mathbb{Q}$ and a function $d_{2}:\left\{x_{\zeta}: \zeta \in \omega_{1}\right\} \rightarrow$ $\mathbb{Q}$ in $V\left[G_{\alpha}\right]$ such that

$$
q_{*} \Vdash^{P_{i} / G_{\alpha}} \forall \zeta \geq \gamma^{*}\left[f\left(\zeta, \dot{\varphi}_{\alpha}\left(x_{\zeta}\right)\right)=d_{1} \dot{\varphi}_{\alpha}\left(x_{\zeta}\right)+d_{2}\left(x_{\zeta}\right)\right]
$$

Thus (working in $\left.V\left[G_{i}\right]\right),\left(h-d_{1} \varphi_{\alpha}\right) \uparrow\left\{x_{\zeta}: \zeta \geq \gamma^{*}\right\}$ belongs to $V\left[G_{\alpha}\right]$ since it equals $d_{2}\left\lceil\left\{x_{\zeta}: \zeta \geq \gamma^{*}\right\}\right.$. But also $\left(h-d_{1} \varphi_{\alpha}\right) \uparrow\left\{x_{\zeta}: \zeta<\gamma^{*}\right\}$ belongs to $V\left[G_{\alpha}\right]$, since $P_{\alpha}$ adds no new countable sequences. Hence $\left(h-d_{1} \varphi_{\alpha}\right) \uparrow\left\{x_{\nu}: \nu \in \omega_{1}\right\}$ belongs to $V\left[G_{\alpha}\right]$ as does $\left(h-d_{1} \varphi_{\alpha}\right) \uparrow K=-d_{1} \psi_{\alpha}$, and therefore, by Lemma 2.5 so does $h-d_{1} \varphi_{\alpha}$.

If $d_{1}=0$, then $h$ belongs to $V\left[G_{\alpha}\right]$ and we are done by induction. If $d_{1} \neq 0$, then since $\left(h-d_{1} \varphi_{\alpha}\right) \uparrow K=-d_{1} \psi_{\alpha}$, we conclude that in $V\left[G_{\alpha}\right], \psi_{\alpha}$ is torsion. But then by definition of the forcing $\varphi_{\alpha}=0$ and hence $h \in V\left[G_{\alpha}\right]$, and again we are done by induction.

\section{Proof of Claim 3.2}

The proof of Claim 3.2 will follow closely along the lines of the proof in 10, but notice the additional universal quantifiers in Claim 4.2 (as compared to [10. Fact G]). The notation $i, q_{*}$ etc. are as in the previous section. We will call a sequence $\bar{\alpha}=$ $\left\langle\alpha_{0}, \ldots, \alpha_{m-1}\right\rangle$ of non-zero ordinals good if $\max \left\{\alpha_{0}, \ldots, \alpha_{m-1}\right\}$ is good in the sense defined after Definition 3.1] (Note that, in contrast to [10], we do not assume that the sequence $\bar{\alpha}$ is increasing.) A sequence $\bar{u}=\left\langle\left\langle a_{k}^{u}, p_{k}^{u}\right\rangle: k\left\langle n^{u}\right\rangle\right.$ is called a candidate if each $p_{k}^{u}$ is a prime and each $a_{k}^{u}$ is a finite non-empty set of ordinals such that for all $k+1<n^{u}$,

$\max \left(a_{k}^{u}\right)<\max \left(a_{k+1}^{u}\right)$. (It is a candidate for initial segments of the functions $a\left(\delta^{*}, \cdot\right)$, $e\left(\delta^{*}, \cdot\right)$ for some $\delta^{*}$.) Given a candidate $\bar{u}$ and $k<n^{u}$, let $\tau_{k}^{u}=\sum\left\{x_{\zeta}: \zeta \in a_{k}^{u}\right\}$. 
Definition 4.1. For any good $\bar{\alpha}$ and candidate $\bar{u}$ and any function $g: \operatorname{rge}(\bar{\alpha}) \rightarrow \omega$, let $T(g, \bar{\alpha}, \bar{u})$ be the set of all functions $t$ from $\left\{\left\langle\alpha_{\ell}, k\right\rangle: \ell<m, g\left(\alpha_{\ell}\right) \leq k<n^{u}\right\}$ to the non-negative integers such that for all $\ell$ and $k, t\left(\alpha_{\ell}, k\right)<p_{k}^{u}$.

If $\bar{\alpha}$ is good and $\bar{u}$ is a candidate, a family $\bar{q}=\left\{q_{t}: t \in T(g, \bar{\alpha}, \bar{u})\right\}$ of conditions in $\tilde{P}_{i}$ is called a $T(g, \bar{\alpha}, \bar{u})$-tree if each $q_{t}$ is stronger than $q_{*}$ and

(a) $q_{t}\left(\alpha_{\ell}\right)\left(\tau_{k}^{u}\right)=t\left(\alpha_{\ell}, k\right)\left(\bmod p_{k}^{u}\right)$ whenever $g\left(\alpha_{\ell}\right) \leq k<n^{u}$;

(b) $q_{t_{1}}\left\lceil\alpha_{\ell}=q_{t_{2}}\left\lceil\alpha_{\ell}\right.\right.$ whenever $t_{1} \uparrow\left(\left\{\alpha_{i}\right\} \times \omega\right)=t_{2}\left\lceil\left(\left\{\alpha_{i}\right\} \times \omega\right)\right.$ for all $\alpha_{i}<\alpha_{\ell}$.

We define $\bar{q} \leq \bar{q}^{\prime}$ if for all $t \in T(g, \bar{\alpha}, \bar{u}), q_{t} \leq q_{t}^{\prime}$.

Claim 4.2. For any $T(g, \bar{\alpha}, \bar{u})$-tree $\bar{q}$, any integers $b_{*}$ and $b_{* *}$, and any countable ordinal $\beta$, there exist $a_{n^{u}}, p_{n^{u}}$, and $\bar{q}^{1}$ such that $p_{n^{u}}>b_{* *}, \bar{u}^{1}=\bar{u} \frown\left\langle a_{n^{u}}, p_{n^{u}}\right\rangle$ is a candidate, $\bar{q}^{1}$ is a $T\left(g, \bar{\alpha}, \bar{u}^{1}\right)$-tree, $\max \left(a_{n^{u}}\right)>\beta$, and

(i) if $s \in T\left(g, \bar{\alpha}, \bar{u}^{1}\right), t \in T(g, \bar{\alpha}, \bar{u})$ and $t \subseteq s$, then $q_{t} \leq q_{s}^{1}$;

(ii) for every $s \in T\left(g, \bar{\alpha}, \bar{u}^{1}\right), q_{s}^{1} \Vdash P_{i} \cdot \dot{h}\left(\tau_{n^{u}}^{u^{1}}\right) \neq b_{*}\left(\bmod p_{n^{u}}\right) "$.

We will prove Claim 4.2 assuming that Claim 3.2 is false. Before doing that, let us see why Claim 4.2 implies a contradiction, thus proving Claim 3.2

Let $N$ be a countable elementary submodel of $\left(H\left(\aleph_{2}\right), \in, P, \Vdash\right)$ such that $N$ is the union $\bigcup_{n \in \omega} N_{n}$ of a chain of elementary submodels such that $\dot{h}, q_{*} \in N_{0}$ and $N_{n} \cap \omega_{1}<$ $N_{n+1} \cap \omega_{1}$ for all $n \in \omega$. Let $\delta^{*}=N \cap \omega_{1}, \delta_{n}=N_{n} \cap \omega_{1}$. We can define by induction on $n \in \omega, g^{n}, \bar{\alpha}^{n}=\left\langle\alpha_{\ell}: \ell<n\right\rangle, \bar{u}^{n}=\left\langle\left\langle a_{\ell}, p_{\ell}\right\rangle: \ell<n\right\rangle$ and $\bar{q}^{n}$ belonging to $N_{n}$ such that for all $n: \bar{q}^{n}$ is a $T\left(g^{n}, \bar{\alpha}^{n}, \bar{u}^{n}\right)$-tree; $g^{n} \subseteq g^{n+1} ; \bar{\alpha}^{n+1}\left\lceil n=\bar{\alpha}^{n} ; \bar{u}^{n+1}\left\lceil n=\bar{u}^{n}\right.\right.$; $\max \left(a_{n}\right)>\delta_{n} ;$ and, denoting $T\left(g^{n}, \bar{\alpha}^{n}, \bar{u}^{n}\right)$ by $T^{n}$ :

(i') if $s \in T^{n+1}, t \in T^{n}$ and $t \subseteq s$, then $q_{t}^{n} \leq q_{s}^{n+1}$;

(ii') for every $s \in T^{n+1}, q_{s}^{n+1} \Vdash^{P_{i}}$ “ $\dot{h}\left(\tau_{n}^{u^{n+1}}\right) \neq n\left(\bmod p_{n}\right) "$; and

(iii') for every $t \in T^{n+1}$ and $\mu \in \operatorname{dom}\left(q_{t}^{n+1}\right), q_{t}^{n+1}(\mu) \in V$ and $\operatorname{dom}\left(q_{t}^{n+1}(\mu)\right) \geq \delta_{n}$; 
and moreover such that every $\zeta \in N \cap i$ equals $\alpha_{n}$ for some $n \in \omega$. It is possible to do this construction by Claim 4.2 using an enumeration of $N \cap i$, since there are arbitrarily large good ordinals $<i$.

By 4.1(b), for each $n \in \omega$ there is $q_{0}^{n} \in Q_{0}$ such that for all $t \in T^{n}, q_{0}^{n}=q_{t}^{n}(0)$. Let $q^{\omega}=\cup_{n \in \omega} q_{0}^{n} \in Q_{0}$, and choose $q^{\prime} \geq q^{\omega}$ in $Q_{0}$ such that

$$
q^{\prime} \Vdash^{Q_{0}} a\left(\delta^{*}, n\right)=a_{n} \wedge e\left(\delta^{*}, n\right)=p_{n} .
$$

We claim that there is an $r \in \tilde{P}_{i}$ such that $\operatorname{dom}(r)=\delta^{*}+1, q^{\prime} \leq r$ and for every $n \in \omega, q_{t_{n}}^{n} \leq r$ for some $t_{n} \in T^{n}$. If so, we have a contradiction because in a model $V[G]$ where $r \in G$ we have: $h\left(z_{\delta^{*}, 0}\right)=n_{o}$ for some $n_{o} \in \omega$, but on the other hand, by (ii'), $h\left(\sum\left\{x_{\zeta}: \zeta \in a\left(\delta^{*}, n_{o}\right)\right) \neq n_{o}\left(\bmod e\left(\delta^{*}, n_{o}\right)\right)\right.$, thus contradicting (2.2).

We will let $r=\cup_{n \in \omega} r^{n}$ where we define by induction $t_{n} \in T^{n}$ and $r^{n}$ such that $r^{n}\left(\alpha_{\ell}\right) \supseteq q_{t_{n}}^{n}\left(\alpha_{\ell}\right)$ for all $\ell<n$. Assuming that $t^{n}$ and $r^{n}$ have been defined for some $n$, we choose

$$
r^{n+1}\left(\alpha_{n}\right) \uparrow\left\{z_{\delta^{*}, k}: k<g^{n+1}\left(\alpha_{n}\right)\right\} \cup\left\{x_{\nu}: \nu \in a\left(\delta^{*}, k\right), k<g^{n+1}\left(\alpha_{n}\right)\right\}
$$

so that the equations (2.4) are satisfied for $\delta=\delta^{*}, k<g^{n+1}\left(\alpha_{n}\right)$ and $q=r^{n+1}\left(\alpha_{n}\right)$. Then we choose $t_{n+1}$ extending $t_{n}$ so that for each $\ell \leq n$, the equations (2.4) are satisfiable for $\delta=\delta^{*}$ and $g^{n+1}\left(\alpha_{\ell}\right) \leq k<n+1$ when $\sum\left\{r^{n+1}\left(\alpha_{\ell}\right)\left(x_{\nu}\right): \nu \in a\left(\delta^{*}, k\right)\right\}=t_{n+1}\left(\alpha_{\ell}, k\right)$ $\left(\bmod p_{k}\right)$. We then let $r^{n+1}\left(\alpha_{\ell}\right)$ agree with $q_{t_{n+1}}^{n+1}\left(\alpha_{\ell}\right)$ on the domain of the latter, for $\ell \leq n$.

There remains the proof of Claim 4.2 assuming that Claim 3.2 is false. We use the notation of Definition 4.1 and Claim 4.2 and let $T=T(g, \bar{\alpha}, \bar{u})$ and $T^{1}=T\left(g, \bar{\alpha}, \bar{u}^{1}\right)$. Let $\alpha_{k}=\max (\bar{\alpha})$; then either (a), (b) or (c) of 3.2 fails for $\operatorname{Pos}_{\alpha_{k}}\left(q_{*}\right)$. Choose $p_{n^{u}}$ larger than 
previous primes, and, if (a) fails and $\left\langle c^{0}, c^{1}, c^{0}, c^{2}\right\rangle$ witnesses the failure, not a divisor of $c^{1}-c^{2}$; if (b) fails and $\left\langle c^{0}, c^{1}, c^{2}, c^{3}, c^{4}, c^{5}\right\rangle$ witnesses the failure, choose $p_{n^{u}}$ not a divisor of $\left(c^{5}-c^{3}\right)\left(c^{2}-c^{0}\right)-\left(c^{3}-c^{1}\right)\left(c^{4}-c^{2}\right)$; if (c) fails and $\left\langle c^{0}, c^{1}, c^{2}, c^{3}\right\rangle,\left\langle c^{4}, c^{5}, c^{6}, c^{7}\right\rangle$ witnesses the failure, choose $p_{n^{u}}$ not a divisor of $\left(c^{3}-c^{1}\right)\left(c^{6}-c^{4}\right)-\left(c^{7}-c^{5}\right)\left(c^{2}-c^{0}\right)$.

Then $T^{1}$ is defined; we must still define $a_{n^{u}}$. Since $T^{1}$ is finite and since it is easy to see that it is possible to choose an $a_{n^{u}}$ such that there are $T^{1}$-trees $\bar{q}^{1}$, it suffices to show that for any fixed node $t_{1}$ of $T^{1}$, any $b_{t_{1}} \in \mathbb{Z}$ and any $T^{1}$-tree $\bar{q}^{1}$ it is possible to choose $\zeta_{t_{1}}^{0}<\ldots<\zeta_{t_{1}}^{s}$ (for some $\left.s=s\left(t_{1}\right)\right)$ such that $\max \left(a_{n^{u}}\right)<\zeta_{t_{1}}^{0}$ and a $T^{1}$-tree $\bar{q}^{\prime} \geq \bar{q}^{1}$ such that (writing $\dot{h}(\zeta)$ instead of $\dot{h}\left(x_{\zeta}\right)$ for clarity of notation) we have:

- for all $t \in T^{1}$ and all $\ell<n^{u}, q_{t}^{\prime}\left(\alpha_{\ell}\right)\left(\sum\left\{x_{\zeta_{t_{1}}^{j}}: j=0, \ldots, s\right\}\right)=0\left(\bmod p_{n^{u}}\right)$;

- for all $t \neq t_{1}, q_{t}^{\prime} \Vdash \dot{h}\left(\sum\left\{\zeta_{t_{1}}^{j}: j=0, \ldots, s\right\}\right)=0\left(\bmod p_{n^{u}}\right)$; and

- $q_{t_{1}}^{\prime} \Vdash \dot{h}\left(\sum\left\{\zeta_{t_{1}}^{j}: j=0, \ldots, s\right\}\right)=b_{t_{1}}\left(\bmod p_{n^{u}}\right)$.

For then we let the new $a_{n^{u}}$ be the union of the old $a_{n^{u}}$ with $\left\{\zeta_{t}^{j}: t \in T^{1}, j=\right.$ $\left.0, \ldots, s\left(t_{1}\right)\right\}$ (for the appropriate choices of $b_{t}$ implying Claim 4.2(ii)). To see how to do this, suppose that for $\alpha_{k}=\max (\bar{\alpha})$, it is case (a) that fails in Claim 3.2 (The other cases are similar.) Suppose that $\left\langle c^{0}, c^{1}, c^{0}, c^{2}\right\rangle \in \operatorname{Pos}_{\alpha_{k}}\left(q_{*}\right)$ with $c^{1} \neq c^{2}$. Let

$$
\begin{aligned}
Z= & \left\{\zeta \in \omega_{1}: \exists r_{1}, r_{2} \in \tilde{P}_{i} \text { s.t. } r_{1}, r_{2} \geq q_{*}, r_{1}\left\lceil\alpha_{k}=r_{2}\left\lceil\alpha_{k},\right.\right.\right. \\
& \left.r_{j}\left(\alpha_{k}\right)\left(x_{\zeta}\right)=c^{0} \text { and } r_{j} \Vdash^{P_{i}} \dot{h}\left(x_{\zeta}\right)=c^{j} \text { for } j=1,2\right\} .
\end{aligned}
$$

Define $\operatorname{Poss}\left(\bar{q}^{1}\right)$ to be the set of all tuples $\left\langle\left(d_{0}^{t}, \ldots, d_{n^{u}-1}^{t}, d_{*}^{t}\right): t \in T^{1}\right\rangle$ such that there exist arbitrarily large $\zeta \in Z$ such that there exists a $T^{1}$-tree $\bar{r} \geq \bar{q}^{1}$ with $r_{t}\left(\alpha_{\ell}\right)\left(x_{\zeta}\right)=d_{\ell}^{t}$ and $r_{t} \Vdash \dot{h}\left(x_{\zeta}\right)=d_{*}^{t}$. As in the argument following Definition 3.1] we can assume that $\operatorname{Poss}\left(\bar{q}^{1}\right)$ is minimal, i.e., not decreased when $\bar{q}^{1}$ increases.

Then there are tuples $\left\langle\left(d_{0}^{t}, \ldots, d_{n^{u}-1}^{t}, d_{*}^{t}\right): t \in T^{1}\right\rangle$ and $\left\langle\left(e_{0}^{t}, \ldots, e_{n^{u}-1}^{t}, e_{*}^{t}\right): t \in T^{1}\right\rangle$ in $\operatorname{Poss}\left(\bar{q}^{1}\right)$ such that $d_{\ell}^{t}=e_{\ell}^{t}$ for all $t, d_{*}^{t}=e_{*}^{t}$ for all $t \neq t_{1}$ and $d_{*}^{t_{1}}=c^{1}, e_{*}^{t_{1}}=c^{2}$. Choose 
$\nu \in \mathbb{Z}$ such that $\left(c^{1}-c^{2}\right) \nu=b_{t_{1}}\left(\bmod p_{n^{u}}\right)$. (This is possible since $c^{1}-c^{2}$ is non-zero in $\mathbb{Z} / p_{n^{u}} \mathbb{Z}$.) Now we can inductively define $\bar{r}^{m+1} \geq \bar{r}^{m} \geq \bar{q}^{1}$ and $\zeta^{m}<\zeta^{m+1}$ in $Z$ for $m<\nu p_{n^{u}}$ such that:

- for $t \in T^{1}$ and $\ell<n^{u}, r_{t}^{m}\left(\alpha_{\ell}\right)\left(\zeta^{m}\right)=d_{\ell}^{t}$;

- for $t \in T^{1}-\left\{t_{1}\right\}, r_{t}^{m} \Vdash \dot{h}\left(\zeta^{m}\right)=d_{*}^{t}$;

- for $m=1\left(\bmod p_{n^{u}}\right), r_{t_{1}}^{m} \Vdash \dot{h}\left(\zeta^{m}\right)=c^{1}$; and

- for $m \neq 1\left(\bmod p_{n^{u}}\right), r_{t_{1}}^{m} \Vdash \dot{h}\left(\zeta^{m}\right)=c^{2}$.

Let $s=\nu p_{n^{u}}$. For $t \in T^{1}$, let $q_{t}^{\prime}=r_{t}^{s}$ and let $a_{n^{u}}=\left\{\zeta^{m}: m \leq s\right\}$. We have:

- $q_{t}^{\prime}\left(\alpha_{\ell}\right)\left(\sum\left\{x_{\zeta^{j}}: j \leq s\right\}\right)=\nu p_{n^{u}} d_{\ell}^{t}=0\left(\bmod p_{n^{u}}\right)$;

- for $t \neq t_{1}, q_{t}^{\prime} \Vdash \dot{h}\left(\sum\left\{\zeta^{j}: \leq s\right\}\right)=\nu p_{n^{u}} d_{*}^{t}=0\left(\bmod p_{n^{u}}\right)$; and

- $q_{t_{1}}^{\prime} \Vdash \dot{h}\left(\sum\left\{\zeta^{j}: \leq s\right\}\right)=\left(c^{1}+\left(p_{n^{u}}-1\right) c^{2}\right) \nu=\left(c^{1}-c^{2}\right) \nu=b_{t_{1}}\left(\bmod p_{n^{u}}\right)$.

\section{Proof of Theorem 0.5}

To prove Theorem 0.5 we use a variation of the forcing defined in section 1: $P^{\prime}=$ $\left\langle P_{i}^{\prime}, \dot{Q}_{i}^{\prime}: 0 \leq i<\omega_{2}\right\rangle$ where $Q_{0}$ is as before and for $i>0, \Vdash_{P_{i}^{\prime}} \dot{Q}_{i}^{\prime}=Q_{\dot{\psi}_{i}}^{\prime}$ for all $i$ (and the enumeration of the names $\left\{\dot{\psi}_{i}: 1 \leq i<\omega_{2}\right\}$ is chosen as before). Let $\varphi_{i} \in V\left[G_{i+1}\right]$ denote the generic function for $Q_{i}$; that is, $\varphi_{i}$ is a homomorphism: $F \rightarrow$ extending $\psi_{i}: K \rightarrow$, where $\psi_{i}$ is the interpretation in $V\left[G_{i}\right]$ of the name $\dot{\psi}_{i}$. Suppose that $\psi_{i}$ represents a torsion element of $\operatorname{Ext}(A, \mathbb{Z})$ in $V\left[G_{i}\right]$ of order $e \geq 1$; that is, there is a homomorphism $\theta_{i}: F \rightarrow \mathbb{Z}$ in $V\left[G_{i}\right]$ such that $\theta_{i}\left\lceil K=e \psi_{i}\right.$. Then $e \varphi_{i}-\theta_{i}: F \rightarrow \mathbb{Z}$ and is identically zero on $K$, so it is a homomorphism from $A$ to $\mathbb{Z}$; we denote it $g_{i}$. (Here, and elsewhere, we shall identify elements of $\operatorname{Hom}(A, \mathbb{Z})$ with homomorphisms from $F$ to $\mathbb{Z}$ which are identically zero on $K$.) If $\psi_{i}$ does not represent a torsion element of $\operatorname{Ext}(A, \mathbb{Z})$, we will let $g_{i}$ be the zero function. 
Let $J=\left\{j \in \omega_{2}: g_{j} \neq 0\right\}$. We will prove that $\operatorname{Hom}(A, \mathbb{Z})$ is free by proving that $\left\{g_{j}: j \in J\right\}$ is a basis of $\operatorname{Hom}(A, \mathbb{Z})$. It is easy to see that this set is linearly independent, since otherwise for some $j_{1}<\ldots<j_{k}$ in $J$ the dependency of $\left\{j_{\nu}: \nu=1, \ldots, k\right\}$ would imply that $\varphi_{j_{k}} \in V\left[G_{j_{k}}\right]$.

To prove that $\left\{g_{j}: j \in J\right\}$ generates $\operatorname{Hom}(A, \mathbb{Z})$ we prove by induction on $i \in \omega_{2}$ that every $h \in \operatorname{Hom}(A, \mathbb{Z})^{V\left[G_{i}\right]}$ is a linear combination of $\left\{g_{j}: j \in J, j<i\right\}$. (Note that every $h \in \operatorname{Hom}(A, \mathbb{Z})^{V[G]}$ belongs to $V\left[G_{i}\right]$ for some $i<\omega_{2}$ since $P$ satisfies the $\aleph_{2}$-chain condition.) The result is true for $i=0$ by Lemma 2.4

Fix $i \in \omega_{2}$ and let $h \in \operatorname{Hom}(A, \mathbb{Z})^{V\left[G_{i}\right]}$. Because we proceed by induction we can suppose that $h \notin V\left[G_{j}\right]$ for any $j<i$; let $q_{*} \in G_{i}$ force this fact. We define $\operatorname{Pos}_{\alpha}\left(q_{*}\right)$ as before and use Claim 3.2

We work in $V\left[G_{\alpha}\right]$. Let $\dot{\varphi}_{\alpha}$ be a $Q_{\alpha}$-name for the generic object given by $Q_{\alpha}$. As in section [3] we can show that there is a rational $d_{1}$ such that $h-d_{1} \varphi_{\alpha}$ belong to $V\left[G_{\alpha}\right]$. Note that $d_{1} \neq 0$ since $h$ does not belong to $V\left[G_{\alpha}\right]$. Since $\left(h-d_{1} \varphi_{\alpha}\right)\left\lceil K=-d_{1} \psi_{\alpha}\right.$, we conclude that in $V\left[G_{\alpha}\right], \psi_{i}$ is torsion, of order $e$ dividing $-d_{1}$; say $d_{1}=n e$. Thus $g_{\alpha}$ is non-zero and equals $e \varphi_{\alpha}-\theta_{\alpha}$ for some $\theta_{\alpha}$ in $V\left[G_{\alpha}\right]$ such that $\theta_{\alpha}\left\lceil K=e \psi_{\alpha}\right.$. Then

$$
h=d_{1} \varphi_{\alpha}+\left(h-d_{1} \varphi_{\alpha}\right)=n g_{\alpha}+n \theta_{\alpha}+\left(h-d_{1} \varphi_{\alpha}\right)
$$

so $h$ equals $n g_{\alpha}$ plus a homomorphism in $V\left[G_{\alpha}\right]$ and by induction we are done. 


\section{REFERENCES}

[1] Devlin, K. J., Shelah, S.: A weak version of $\diamond$ which follows from $2^{\aleph_{0}}<2^{\aleph_{1}}$, Israel J. Math. 29, 239-247 (1978)

[2] Eklof, P. C., Huber, M.: On the rank of Ext, Math. Zeit. 174, 159-185 (1980)

[3] Eklof, P. C., Mekler, A. H.: Almost Free Modules, (North-Holland Math. Library v. 46) Amsterdam New York Oxford Tokyo: North-Holland 1990

[4] Eklof, P. C., Shelah, S.: A non-reflexive Whitehead group, to appear in J. Pure and Appl. Algebra.

[5] L. Fuchs, Infinite Abelian Groups, vol. I (Pure and Applied Mathematics v. 36), New York London: Academic Press 1970

[6] Golasiński, M., Gonçalves, D. L.: On co-Moore spaces, Math. Scand. 83, 42-52 (1998)

[7] Gregory, J.: Higher Souslin trees and the generalized continuum hypothesis, J. Symbolic Logic 41, 663-671 (1976)

[8] Hiller, H., Huber, M., Shelah, S.: The structure of $\operatorname{Ext}(A, \mathbf{Z})$ and $V=L$, Math. Z. 162, 39-50 (1978)

[9] Nunke, R. J., Rotman, J. J.: Singular cohomology groups, J. London Math. Soc., 37, 301-306 (1962)

[10] Shelah, S.: The consistency of $\operatorname{Ext}(G, \mathbb{Z})=\mathbb{Q}$, Israel J. Math. 39, 74-82 (1981)

[11] Shelah, S.: Models with second order properties, III. Omitting types for L(Q), Arch. Math. Logik 21, 1-11 (1981)

(Eklof) MATh DePt, UCI, IRvine, CA 92697-3875

(Shelah) Institute of Mathematics, Hebrew University, Jerusalem 91904, Israel 